\title{
Likelihood ratio evaluation of $M$-state models applied to individual choice sequences of successive reversal learning'
}

WILLIAM F. LOWE. JR.. THE UNIVERSITY OF TEXAS, Austin, TeX., AND JOHN THEIOS, THE UNIVERSITY OF WISCONSIN, Madison, Wis. 53706

The response sequences of 12 rats given 35 reversals of a position discrimination were analyzed by likelihood ratio analyses in order to determine the minimum number of response probability values (states of responsiveness) necessary to adequately account for the individual response sequences. It was found that a two-state process could account for the data of all the reversals but the first one.

Consider an experimental situation in which the $\mathrm{S}$ is required to make a series of binary responses to a recurring stimulus Situations of this type would include typical learning experiments in which responses are classed as either correct or incorrect. There have been a considerable number of attempts to describe data of this type within the theoretical framework of homogeneous Markov processes. Typically, the investigator decides on a particular model and then attempts to show that it is a good model in terms of the closeness of the fit of predictions of various statistics such as the distributions of errors before the first success, total errors and trial of the last error. It is possible, however, to formulate and test a nested series of hypotheses concerning the response sequence of an individual $S$ in this type of experiment First, it could be assumed that all the responses were generated while the organism was in one state of responsiveness. This hypothesis might be appropriate for responding at an asymptotic level. An alternative hypothesis is that the responses were generated while the organism was in two states of responsiveness. Similar hypotheses can be formulated to include any number of states of responsiveness.

Using a technique described by Theios (1967), it is possible to easily obtain maximum likelihood estimates of the parameters of an inhomogeneous Markov process with any finite number of states. For the two-state Bower (1961) type models if it is assumed that the $S$ starts in State $S_{1}$, and with probability $c_{n}$ may make a transition on any trial $n$ to $S$ tate $S_{2}$, then a posteriori there exists an integer, $i$, which designates the trial on which the transition in fact took place. Given the transition trial integer, $i$, the likelihood of a response sequence $N$ trials long for a single $S$ is

$$
\mathrm{L}_{2}=\left(\hat{\mathrm{p}}_{1}\right)^{\mathrm{x}}\left(1-\hat{\mathrm{p}}_{1}\right)^{\mathrm{i}-\mathrm{x}}\left(\hat{\mathrm{p}}_{2}\right)^{\mathrm{y}}\left(1-\hat{\mathrm{p}}_{2}\right)^{\mathrm{N}-\mathrm{i}-\mathrm{y}}
$$

where $\hat{p}_{1}$ and $\hat{p}_{2}$ are maximum likelihood estimates of $\mathrm{p}_{1}$ and $\mathrm{p}_{2}$ (the probability of a successful response in the first and second states, respectively), $x$ is the number of successes in the first $i$ trials, and $y$ is the number of successes in the last $\mathrm{N}-\mathrm{i}$ trials. If $i$ is known, then the problem of obtaining maximum likelihood estimates of the parameters is trivial; $\hat{p}_{1}$ is just the proportion of successes in the first $i$ trials and $\hat{p}_{2}$ is the proportion of successes on all trials from $i+1$ to $N$. All of these values can be calculated from the $S$ 's sequence of responses.

Given the likelihood equation, it is possible to select the integer i so that the likelihood function is maximized. Similar analyses can be made for any M-state Markov model, where $M$ can take on the values $1,2,3, \cdots$. State transitions then are assumed to take place on $\mathrm{M}-1$ trials $\mathrm{i}, \mathrm{j}, \mathrm{k}, \cdots$ which maximize the likelihood function. Once the maximized likelihood functions of several models are obtained, it is possible to utilize a likelihood ratio criterion to determine the minimum number of states necessary to describe the response sequence under consideration. Let $L_{g}$ and $L_{h}$ represent the maximum likelihoods of a sequence of responses under the hypothesis that the responses are generated by processes with $g$ and $h$ states, respectively, $h>g$. If the responses really were generated by a g-state process, then $-2 \log _{e}\left(\mathrm{~L}_{\mathrm{g}} / \mathrm{L}_{\mathrm{h}}\right)$ has' a distribution which is approximated by the $x^{2}$ distribution with [ $\left.3(\mathrm{~h}-\mathrm{g})-1\right]$ degrees of freedom. If the transformed likelihood ratio is significantly large then there is sufficient evidence to reject the hypothesis that the response se- quence was generated by a process isomorphic to the g-state as compared to the h-state model.

Method. The likelihood ratio analysis just described was applied to the data of a successive reversal experiment in order to determine the minimum number of probability values, or states, necessary to describe the response sequences. Twelve rats were given 35 reversals of a two-choice position problem in a Skinner box with two retractable bars. The trials were discrete with a $30 \mathrm{sec}$ intertrial interval. A noncorrection procedure was used. The reward outcome was three $45 \mathrm{mg}$ Noyes pellets for each correct bar press. Each day, the Ss were given 10 trials on the previous day's problem followed by 50 trials on which the response-outcome contingencies were the opposite of those of the previous day.

Results and Discussion. The learning data were similar to that found in previous reversal studies (North, 1950; Dufort, Guttman, \& Kimble, 1954; Gonzales, Roberts, \& Bitterman, 1964). Statistics such as the trial of the last error, number of errors before the first success and total errors decreased as the number of reversals increased. All of these statistics appeared to reach stable values by reversal 25 and remained relatively constant thereafter.

As indicated above, it is possible to determine the minimum number of states a model need have to satisfactorily describe the reversal data. Four models were tested, a four-state Markov process, a three-state process, a two-state process, and a single state process. The analysis involved selection of the appropriate transition integers to maximize the likelihood function for the response sequence of each individual $\mathrm{S}$. Each reversal was analyzed separately. Presumably, if a many state process were involved in the early reversals, the four-state model would be significantly better than the three- or two-state model, as evaluated by the likelihood ratio criterion. Similarly, it was expected that as the number of reversals increased, the Ss would more rapidly learn the discrimination, and a two-state model would be more appropriate. Suprisingly, it was found that the two-state model accounted for the response sequences of both the early and the later reversals as well as any of the models with more states. Figure 1 shows the mean maximum likelihood taken over all Ss for the first and last five reversals. As can be seen, there is little difference between the likelihood values of the two-, three- or four-state models in either

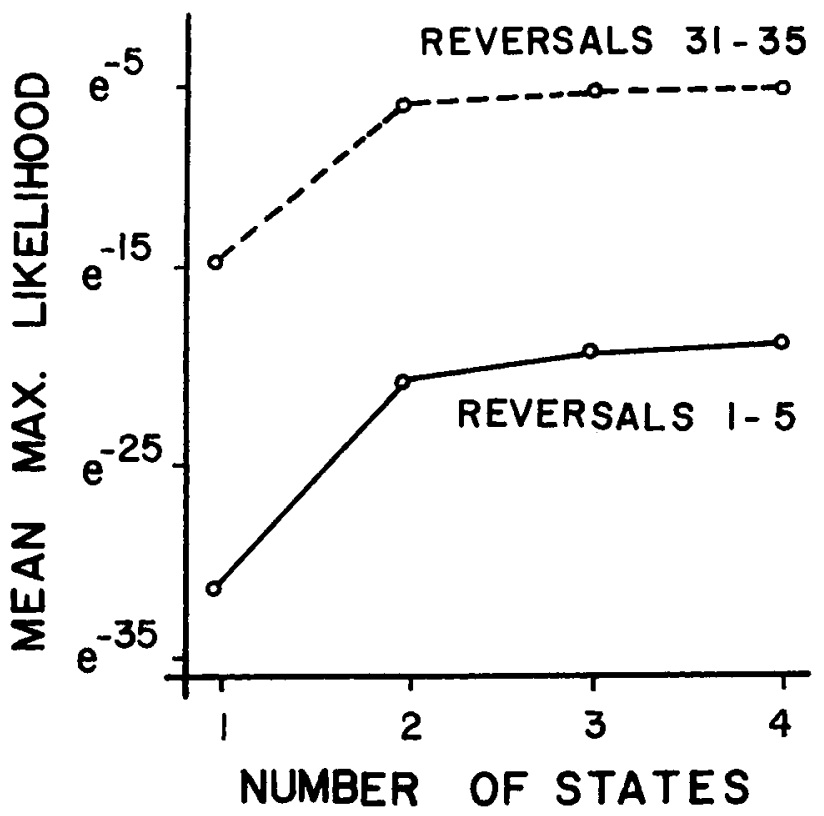

Fig. 1. Group mean maximum likelihood values for reversals 1 through 5 and reversals 31 through 35 . 


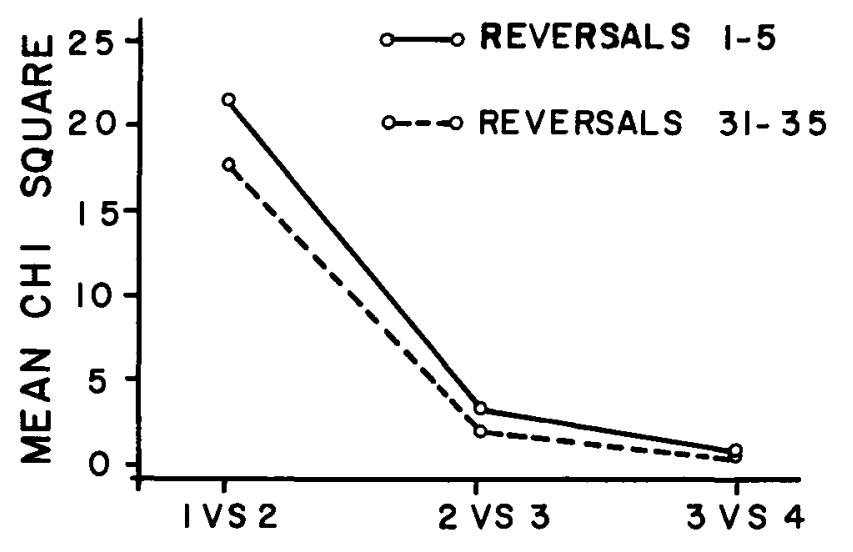

STATES

Fig. 2. Group mean chi square approximations obtained in pairwise likelihood ratio tests for reversals 1 through 5 and reversals 31 through 35 (df $=2$ in each case).

case. The lack of differences between the two-, three- and four-state models is also shown in Fig. 2, which gives the mean chi square approximations yielded by the likelihood ratio transformation. It was not possible to reject the hypothesis that the data were generated by a process isomorphic with a two-state model, as tested against a three-state model. In fact, the three-state model was found to be significantly better than the two-state model only for the first reversal.

The increase in the likelihood values from the first five reversals to the last five reversals is a result of the average trial of the transition decreasing from about 25 to 5 between these reversals.
Over the 35 reversals, the mean of the estimates of the trial of transition decreased geometrically, and approached the value of the mean trial of the last error. The mean of the estimates of the asymptotic response probability, $\hat{\mathbf{p}}_{2}$, increased slowly from a value of .80 and approached a value of 1.00 over the 35 reversals. On the other hand, the mean of the estimates of the initial response probability, $\hat{p}_{1}$, remained relatively stationary over the 35 reversals at a value of about .20 . This is what would be expected on the first few reversals. However, the lack of a decrease in $\hat{p}_{1}$ over reversals presumably was due to the rats learning to anticipate the reversal since it always came after 10 trials with the previous day's response-outcome contingency.

On the basis of the results of Dufort, Guttman, \& Kimble (1954), who found one-trial reversals in a similar situation, it would be expected that if training were continued, the transition integer value for the two-state model and the value of the trial of the last error would be equal and eventually drop to unity, the case where animals are making one-trial reversals.

\section{REFERENCES}

BOWER, G. H. Application of a model to paired-associate learning. Psychometrika, 1961, 26, 255-280.

DUFORT, R. H., GUTTMAN, N., \& KIMBLE, G. A. One-trial discrimination reversal in the white rat. J. comp. physiol. Psychol., 1954, 47, 248.249.

GONZALES, R. C., ROBERTS, W. A., \& BITTERMAN, M. E. Learning in adult rats with extensive cortical lesions made in infancy. Amer. J. Psychol., 1964, 77, 547-562.

NORTH, A. J. Improvement in successive discrimination reversals. J. comp. physiol. Psychol., 1950, 43, 442-460.

THEIOS, J. Finite integer analysis of individual learning sequences. Technical Report No. 14, Conditioning Research Laboratory, Department of Psychology, University of Texas, 1967.

\section{NOTE}

1. This research was supported in full by Public Health Service Research Grants HD 02212-02 and HD 03540-01. 